\title{
Hypothalamo-Pituitary Innsufficiency Presenting With Acute Kidney Injury
}

\author{
Can Hüzmeli ${ }^{1, *}$, Özcan Demetgül ${ }^{2}$, Eda Payasılı ${ }^{3}$ Erol Öztürk ${ }^{4}$, Bestami Barıs Celik ${ }^{5}$, \\ Ali Borazan ${ }^{1}$ \\ ${ }^{1}$ Department of Nephrology, Hatay Education and Research Hospital, Hatay, Turkey. \\ ${ }^{2}$ Department of Neurology, Hatay Education and Research Hospital, Hatay, Turkey. \\ ${ }^{3}$ Department of Radiology, Hatay Education and Research Hospital, Hatay, Turkey. \\ ${ }^{4}$ Department of General Surgery, Private Academy Hospital, Hatay, Turkey. \\ ${ }^{5}$ Department of Anesthesia and Reanimation, Hatay Education and Research Hospital, Hatay, Turkey.
}

How to cite this paper: Can Hüzmeli, Özcan Demetgül, Eda Payaslı Akbelen, Erol Öztürk, Bestami Barıs Celik, Ali Borazan. (2022) Hypothalamo-Pituitary Innsufficiency Presenting With Acute Kidney Innjury. International Journal of Clinical and Experimental Medicine Research, 6(2), 122-124.

DOI: $10.26855 /$ ijcemr.2022.04.003

Received: December 27, 2021

Accepted: January 23, 2022

Published: February 16, 2022

*Corresponding author: Can Hüzmeli, Department of Nephrology, Hatay Education and Research Hospital, Antakya, Hatay, Turkey.

Email: chuzmeli@hotmail.com

\begin{abstract}
Diabetes insipidus (DI) is a rare disorder of water homeostasis and characterized by the persistent excretion of abnormally large of volumes of dilute urine. DI is a syndrome characterized by polyuria, polydipsia. DI can be divided into four forms, central DI, nephrogenic DI, gestational DI, and primary polydipsia. Central and nephrogenic DI may be acquired or inherited. Central diabetes insipidus (DI) is a rare endocrine disorder. Central DI results from a defect in arginine vasopressin (AVP) production, while nephrogenic DI results from a defect in AVP renal response. The most common treatment of Central DI is the administration of synthetic ADH, desmopressin. Dehydration may develop in these patients. Hypernatremia and rhabdomyolysis may also occur. As a result, acute kidney injury occurs in some cases. In this article, we tried to discuss a case of hypothalamo-pituitary insufficiency in a 47-year-old female patient who presented with acute kidney injury, in the light of the literature.
\end{abstract}

\section{Keywords}

Central diabetes insipidus, Hypernatremia, dehydration, Acute kidney injury

\section{Introduction}

Diabetes insipidus (DI) is characterized by polyuria and polydipsia due to insufficient secretion of arginine vasopressin (AVP) or impaired renal response to AVP. DI is a disorder of the water balance. The water balance is maintained by the AVP, the thirst mechanism and the kidneys. It also increases the risk of volume reduction and hypernatremia in cases of markedly increased thirst and urination [1].

AVP or antidiuretic hormone (ADH) is a small peptide hormone produced by magnocellular neurons in the supra-optic and para-ventricular nuclei of the hypothalamus and reflected to the posterior pituitary via the stem (1). Release and synthesis of AVP are regulated mainly by plasma osmolality (or serum $\mathrm{Na}$ ) under physiological conditions. DI is a rare disease that affects about 1 in 25,000 people. DI can be classified into four main categories: central, nephrogenic, dipsogenic, or gestational. The most common type, central DI, is caused by a deficiency in $\mathrm{ADH}$ production, usually caused by damage to the pituitary gland. DI is most commonly defined as a urine volume greater than 3-3.5 liters over a 24-hour period in adults with a urine osmolality below $300 \mathrm{mOsmol} / \mathrm{kg}$ and increased serum osmolality [2]. 


\section{Case}

A 47-year-old woman was referred to the Neurology outpatient clinic from Syria with progressive gait disturbance that started about a year ago. The patient had not been able to walk for about 6 months. She had a history of type 2 diabetes mellitus and hypothyroidism, was using insulin and levothyron, and her family history showed no feature. In physical examination; Her general condition was moderate, she was conscious, cooperative oriented, dysarthric speech, prone to sleep, blood pressure was 90/50 $\mathrm{mmHg}$, heart rate was 65/min, 0/5 loss of strength in the lower extremities (paraplegic). Laboratory findings made on 13.08.2021; glucose 115mg/dl (70-110), blood urea nitrogen (BUN) 14mg/dl (7-25), serum creatine 0.9mg/dl $(0.4-1.1 \mathrm{mg} / \mathrm{dl})$, calcium 10mg/dl 8.6-10), phosphorus 4.8mg/dl (2.7-4.5), serum creatinine kinase 109 IU/L (22-200), sodium 148mmol/L (135-145), potassium $5.5 \mathrm{mmol} / \mathrm{L}$ (3.5-5.5), the patient was admitted to the neurology service with the preliminary diagnosis of acute polynoropathy. EMG revealed mixed type polyneuropathy.

On 23.08.2021, the patient was admitted to our service with acute kidney injury (AKI) after the following analysis; glucose $114 \mathrm{mg} / \mathrm{dl}$, BUN $21.5 \mathrm{mg} / \mathrm{dl}$, serum creatine $3.2 \mathrm{mg} / \mathrm{dl}$, serum uric acid $11.3 \mathrm{mg} / \mathrm{dl}$ (2.6-6), sodium $173 \mathrm{mmol} / \mathrm{L}$ (plasma osmolality $359 \mathrm{mOsm} / \mathrm{kg}$ ), potassium $3.8 \mathrm{mmol} / \mathrm{L}$ calcium $9.9 \mathrm{mg} / \mathrm{dl}$, phosphorus $3.8 \mathrm{mg} / \mathrm{dl}$, TSH 2.8mU/L (0.27-4.2), FT3 2.4ng/dl (2-4.4), FT4 $0.45 \mathrm{ng} / \mathrm{dl}$ (0.93-1.7) and a urine density of 1006 in the complete urinalysis. It was thought that the patient might have DI after it was determined that the patient took 1500cc and removed 3000cc. In addition, the patient's thyroid hormones were evaluated as central hypothyroidism. The patient's pituitary hormones were requested, cortisol $1.2 \mathrm{ug} / \mathrm{dl}$ (6.2-19.4), ACTH 1pg/ml (7.2-63), LH 0.44 U/L (3-12) and FSH 2, 2U/L (2-6.6), GH 0.29ng/ml (0-5) and ADH 2.54pmol/L were detected. The patient was started on hydration. The patient, who was evaluated as central DI, was started on minirin 60mcg twice a day and methylprednisolone. After the 3rd day of treatment, levatirone was added to the treatment. Post-treatment laboratory values, on 20.09 .2021 were BUN $11.7 \mathrm{mg} / \mathrm{dl}$, serum creatinine $0.9 \mathrm{mg} / \mathrm{dl}$, serum uric acid $5.5 \mathrm{mg} / \mathrm{dl}$, sodium $140 \mathrm{mmol} / \mathrm{L}$, potassium $3.9 \mathrm{mmol} / \mathrm{L}$, complete urinalysis urine density was detected as 1011 . The patient's minirin dose was reduced.

The general condition of the patient improved, first of all her speech improved, and then the patient began to walk slowly. In the patient's pituitary magnetic resonance imaging; In the posterior part of the optic chiasm, a mass lesion of approximately $13 \times 10 \times 10 \mathrm{~mm}$, extending to the level of the interpeduncular cistern, showing contrast enhancement was detected. After the patient stopped taking minirin on 01.10.2021, AKI and hypernatremia developed again after the patient worsend (BUN 45mg/dl, serum creatine 1.6mg/dl, serum uric acid 10.2mg/dl and sodium $167 \mathrm{mmol} / \mathrm{L}$ ). After the drug was started again, the patient's biochemical values returned to normal and her complaints disappeared.

\section{Discussion}

Central DI is caused by a deficiency of ADH, an ADH that acts on V2 receptors in the kidney to promote free water reabsorption. There are three types of central DI, known as idiopathic, secondary, and familial. The most common cause of central DI is tumors in the central nervous system. It can also develop as a result of inflammatory diseases, surgical interventions, head trauma and autoimmune diseases. Although rare, central DI also occurs genetically. This subclass of central DI is called familial neurohypophysial diabetes insipidus, which is mostly inherited in an autosomal dominant mode. AVP deficiency in the central DI is associated with chronic low uric acid clearance and hyperuricemia [3-4].

Lima et al. reported a case of acute kidney injury (serum creatinine $5.4 \mathrm{mg} / \mathrm{dl}$ ) and hypernatremia (serum sodium $221 \mathrm{mEq} / \mathrm{l}) 25$ days after aneurysm surgery in a 53-year-old male patient. DI-induced hypernatremia developed in the case, and rhabdomyolysis (creatine phosphokinase 253,200IU/L) due to hypernatremia was detected. AKI developed as a result of hypernatremia and rhabdomyolysis. It has been demonstrated to improve with desmopressin therapy [5]. Kung et al. reported a case of hypernatremia (serum sodium $187 \mathrm{mmol} / \mathrm{L}$,) due to DI (increased serum osmolality and decreased urine osmolality) and rhabdomyolysis due to hypernatremia (creatine phosphokinase 60,720IU/L) in a 23-year-old male patient. In this case, germinoma was detected and treated with desmopressin [6]. DI and oliguric acute kidney injury developed in a 19-year-old male patient who had a head injury. HD was taken from the patient, then urine output increased, and serum creatinine value returned to normal. In the follow-up, urine output was 23lt and 25lt, low urine density $(1,000-1,003)$ and low urine osmolality were detected. The patient was diagnosed with central DI and was treated with desmopressin [7].

Hypernatremia can lead to AKI as a result of dehydration and/or rhabdomyolysis. Therefore, regular monitoring 
of kidney function and muscle enzymes is also recommended in patients with DI. In the treatment of central DI, it is necessary to replace the lack of free water with appropriate fluid intake, start desmopressin, the synthetic analogue of the missing hormone AVP, and treat the underlying condition. Desmopressin is a synthetic analogue of vasopressin that has a prolonged action profile with minimal vasopressor activity. Treatment with desmopressin mainly targets relief of polyuria, nocturia and associated thirst symptoms. Desmopressin can be administered orally, intranasally, subcutaneously, or intravenously. The serum sodium level should be corrected at a rate of 0.5 $\mathrm{mEq} / \mathrm{L} /$ hour without a decrease of more than $8-10 \mathrm{mEq} / \mathrm{L}$ in 24 hours, the target sodium level should be determined as $145 \mathrm{mEq} / \mathrm{L}[8-9]$.

\section{Conclusion}

As a result, patients with DI may develop acute kidney injury as a result of dehydration due to hypernatremia or rhabdomyolysis. It would be appropriate to evaluate for DI in patients presenting with acute kidney injury and hypernatremia.

\section{Acknowledgements}

We thank the patient and his son for permission to publish. We also thank the Internal Medicine specialist Umut Eraslan, who referred this patient to our clinic.

\section{References}

[1] Priya, G., Kalra, S., Dasgupta, A., and Grewal, E. (2021). Diabetes insipidus: A pragmatic approach to management. Cureus, 13(1).

[2] Mutter, C. M., Smith, T., Menze, O., Zakharia, M., and Nguyen, H. (2021). Diabetes Insipidus: Pathogenesis, Diagnosis, and Clinical Management. Cureus, 13(2).

[3] Verbalis, J. G. (2020). Acquired forms of central diabetes insipidus: mechanisms of disease. Best Practice \& Research Clinical Endocrinology \& Metabolism, 101449.

[4] Arima, H., Azuma, Y., Morishita, Y., and Hagiwara, D. (2016). Central diabetes insipidus. Nagoya Journal of Medical Science, 78(4), 349.

[5] Lima, E. Q., Aguiar, F. C., Barbosa, D. M., and Burdmann, E. A. (2004). Severe hypernatraemia (221 mEq/l), rhabdomyolysis and acute renal failure after cerebral aneurysm surgery. Nephrology Dialysis Transplantation, 19(8), 2126-2129.

[6] Kung, A. W., Pun, K. K., Lam, K. S., and Yeung, R. T. (1991). Rhabdomyolysis associated with cranial diabetes insipidus. Postgraduate Medical Journal, 67(792), 912-913.

[7] Ulu, S. M., Polat, M., Altug, A., and Yuksel, S. (2014). A fatal situation: Diabetes insipidus and superimposed acute polyuric kidney injury. Elective Medicine Journal, 2(3), 321.

[8] Garrahy, A., and Thompson, C. J. (2020). Management of central diabetes insipidus. Best Practice \& Research Clinical Endocrinology \& Metabolism, 34(5), 101385.

[9] Almalki, M. H., Ahmad, M. M., Brema, I., Almehthel, M., AlDahmani, K. M., Mahzari, M., and Beshyah, S. A. (2021). Management of Diabetes Insipidus following Surgery for Pituitary and Suprasellar Tumours. Sultan Qaboos University Medical Journal, 21(3), 354. 